Research Article

\title{
Knowledge, Attitudes, and Practices about Trachoma in Rural Communities of Tigray Region, Northern Ethiopia: Implications for Prevention and Control
}

\author{
Hailay Gebretnsae $(\mathbb{D}$, Nega Mamo, Tesfay Teklemariam, Kiros Fenta $(\mathbb{D}$, \\ Tesfay Gebrehiwet, Abera Berhe $\mathbb{D}$, Fana Gebreselasie, and Kiros Demoz
}

Tigray Health Research Institute, Mekelle, Tigray, Ethiopia

Correspondence should be addressed to Hailay Gebretnsae; hailish14@gmail.com

Received 12 March 2020; Revised 4 June 2020; Accepted 22 June 2020; Published 25 July 2020

Academic Editor: Pam R. Factor-Litvak

Copyright (c) 2020 Hailay Gebretnsae et al. This is an open access article distributed under the Creative Commons Attribution License, which permits unrestricted use, distribution, and reproduction in any medium, provided the original work is properly cited.

\begin{abstract}
Background. Trachoma is a neglected tropical disease which is the leading infectious cause of blindness in the world. Trachoma is one of the major health problems in Tigray Region, Northern Ethiopia. However, knowledge, attitudes, and practices about trachoma are not yet studied in depth. The objective of the study was to assess knowledge, attitudes, and practices on trachoma and its associated factors among rural communities in two districts of Tigay Region, Northern Ethiopia. Methods. A cross-sectional study was conducted in two districts of Tigray Region, Northern Ethiopia, from May 7-24, 2017. Data were collected on paper based, were entered into Epi Info version 3.5.1, and then exported to SPSS version 21 for analysis. Logistic regression analysis was done to identify factors associated with knowledge, attitudes, and practices. Results. In this study, a total of 194 respondents were included. The overall level of good knowledge, attitudes, and practices on trachoma was $51 \%, 49.5 \%$, and $35.6 \%$, respectively. Having ever received health education was significantly associated with good knowledge (adjusted odds ratio (AOR) $=4.10$; $95 \%$ confidence interval (CI): 1.91-8.79) and attitudes (AOR =2.10; 95\% CI: 1.02-4.25). Moreover, good knowledge was associated with good practices on trachoma prevention and control ( $\mathrm{AOR}=2.86 ; 95 \% \mathrm{CI}$ : $1.46-5.62)$. Conclusion. Our study implies that areas with high burden of trachoma need to improve communities' knowledge, attitudes, and practices towards trachoma prevention and control in order to eliminate trachoma as a public health problem. Therefore, health education focused on SAFE strategy should be provided to increase knowledge and changing attitudes that contribute for good practices towards trachoma prevention and control among communities.
\end{abstract}

\section{Introduction}

Trachoma is a neglected tropical disease which is the leading infectious cause of blindness in the world [1-3]. Trachoma largely affects people in low socioeconomic conditions in the rural areas and usually communities with poor hygiene and environmental sanitation [2]. In 2019, globally, the number of people at risk of trachoma was 142.2 million and it is responsible for the visual impairment of about 1.9 million people. Ethiopia is one of the most trachoma-affected countries which shares about $50.6 \%$ of the burden in the world $[4,5]$.
In 1998, the World Health Organization (WHO) formally adopted the SAFE strategy (surgery, antibiotics, facial cleanliness, and environmental improvements) for the effective prevention and control of trachoma [3]. The target for eliminating trachoma as a public health problem can be achieved if the SAFE strategy is properly applied. However, trachoma still remains one of the major health problems in the world [4].

Several factors are associated with increased risk of trachoma. These include lack of water, poor personal hygiene, and environmental sanitation [6-8]. Furthermore, poor knowledge $[9,10]$, and unfavorable sociocultural 
perceptions and poor practices about prevention of trachoma are the main factors in the transmission and sustaining of the infection of trachoma in the communities [11-14].

Trachoma is highly endemic in Tigray Region, Northern Ethiopia, especially in Raya-Alamata and Endamekoni districts (the study area). In 2013, the adjusted prevalence of the clinical sign trachomatous inflammation-follicular (TF) among children aged 1-9 years in the enumeration units encompassing Raya-Alamata and Endamekoni districts was $41.4 \%$ and $41.0 \%$, respectively [15]. However, there are no data on knowledge, attitudes, and practices on trachoma prevention and control among rural communities in the two districts so far. Therefore, areas with high burden of trachoma such as Alamata and Endamekoni districts need to assess knowledge, attitudes, and practices and its associated factors of trachoma among rural communities. The findings obtained from the study are of paramount importance for health providers and program managers in planning and implementing to improve prevention and control of trachoma among communities. Moreover, it is useful to policy makers to formulate appropriate interventions for implementation of the SAFE strategy.

\section{Methods}

2.1. Study Design and Area. A community based crosssectional study design was conducted to assess knowledge, attitudes, and practices on trachoma among rural communities in two districts (Raya-Alamata and Endamekoni) of Tigray Region, Northern Ethiopia, from May 7-24, 2017. According to the 2007 census, the total estimated population was 96,132 in Raya-Alamata and 94,635 in Endamekoni districts in 2017. From 2014 to 2018; LIGHT FOR THE WORLD in collaboration with the Tigray Regional Health Bureau (TRHB) had a project to eliminate trachoma as a public health problem in the two districts. The focusing activities of the project were mainly on communities' awareness creation and behavioral change to improve hygiene and environmental sanitation by using different communication channels and health education. The Zithromax MDA campaign was carried out in April 2017 (fourth round) before three-four weeks of the data collection in both districts.

2.2. Sample Size Determination. The sample size was calculated using single population proportion formula with the assumption of proportion of had heard about trachoma among communities in rural Amhara Region, Ethiopia, which was $85.6 \%$ [16], using 5\% margin of error, $95 \%$ confidence level, and nonresponse rate of $10 \%$, and the total sample size was 209.

2.3. Sampling Procedures. Two districts were purposively included in the study. We randomly selected four kebelles (the smallest administrative unit) by the lottery method from the selected districts (2 kebelles from each district). The list of all Women Development Groups (WDGs) with their respective list of households in each selected kebelle was obtained from the health post WDG registration books, and then six WDGs were selected within each selected kebelle using the lottery method. (The WDG is the smallest community organization unit and consists of groups of 25-30 households residing in a neighborhood). Finally, 209 households in the selected WDGs were enrolled using systematic random sampling technique.

2.4. Data Collection Procedures and Data Quality Assurance. A structured questionnaire was adapted from a previous study [16], after reviewed the different related literature. Data were collected by face-to-face interviews and by observations for some practice-related questions through a paper-based questionnaire. We interviewed mothers of households about sociodemographic information, knowledge, attitudes, and practices on trachoma, Zithromax uptake, water, sanitation, and hygiene (WASH), health education, and promotion. Their responses on some practice-related questions were verified by observational technique (availability of latrine and utilization, house compound cleanliness, having separated human and animal dwellings, and solid and liquid waste disposal methods). Six diploma nurses to collect data and two bachelor-degree health officers to supervise the data collection process were recruited. Data collectors and supervisors were trained for two days on the questionnaire, data collection techniques, and the purpose of the study. The pretest was conducted in $5 \%$ of respondents (10 households) in two kebelles other than the study sites. Daily completeness and checking for missing data was done by the data collectors and supervisors.

2.5. Operational Definition. Knowledge, attitudes, and practices on trachoma were assessed using 12 knowledgerelated, 4 attitude-related, and 8 practice-related questions on trachoma. Correct answers were given a score of one, incorrect answers were scored zero, and those who scoring greater than the mean were considered to have good knowledge, attitudes, and practices, respectively.

2.6. Ethical Approval. The study protocol was reviewed and approved by the institutional ethical review committee of Tigray Health Research Institutes (reference number: THRI/ RM012/2017). The support letter was obtained from the Tigray Regional Health Bureau and the selected district health offices. The respondents were informed about the objective and purpose of the study, and verbal consent was obtained. Confidentiality of the information was assured, and participants were informed that they have the right to withdraw from the interview at any stage.

2.7. Data Processing and Analysis. Data were entered into Epi Info version 3.5.1 and then imported to SPSS version 21 for analysis. Data cleaning and editing was carried out before analysis. Knowledge, attitudes, and practices were computed separately, and its total scores were recorded in to dichotomous categorical variables (good vs poor) based on their 
mean score stated in operational definition. Frequency distribution and percentages were performed using frequency tables. Bivariate logistic regression analysis was done for each outcome variables (knowledge, attitudes, and practices). All variables with a $p$ value $<0.25$ in the bivariate analysis were included in the multivariable logistic regression model. Finally, variables which had a $p$ value with less than 0.05 were considered statistically significant.

\section{Results}

3.1. Sociodemographic Characteristics of Study Participants. A total of 194 participants were included in this study, and this made a response rate of $92.8 \%$. The remaining $(7.2 \%)$ participants were not included due to closed households during the data collection period. From a total respondents, $87(44.8 \%)$ were in the age range of 30-49 years and 112 $(57.7 \%)$ were currently in the union of marital status. Majority of the respondents, 180 (92.8\%), were house wife and $155(79.9 \%)$ were illiterate (Table 1$)$.

3.2. Knowledge on Trachoma. Most (89.2\%) of respondents had ever heard about trachoma. Above half (54.6\%) of respondents knew that trachoma can be transmitted from person to person and answered correctly that trachoma can be transmitted by dirty fingers (53.1\%), flies (35.6\%), and by using contaminated towel (24.7\%). The majority, 164 $(84.5 \%)$, of respondents knew trachoma as a preventable disease, and $161(83 \%)$ of respondents knew that trachoma can lead to blindness. The mean score of respondents on knowledge was $6.79 \pm 3.29$ standard deviation (SD). Ninetynine $(51 \%)$ of respondents were scored above the mean score and classified as having good knowledge on trachoma (Table 2).

3.3. Attitudes on Trachoma Prevention and Control. Most; 185 (95.4\%), of participants agreed availability of adequate water is important for trachoma prevention, and $171(86.1 \%)$ of respondents believed taking mass drug administration is important to prevent and control trachoma. Overall, 96 $(49.5 \%)$ of respondents were classified as having good attitudes on trachoma prevention and control with a mean score of $3.39 \pm 0.69$ SD (Table 3 ).

3.4. Practices on Trachoma Prevention and Control. The majority, 171 (88.1\%), of respondents took mass drug administration, and 149 (76.8\%) of households had separated human and animal dwellings. However, of the total 194 households, only $72(37.1 \%)$ were utilizing a latrine and 59 (30.4\%) had clean house compounds. Out of the total, 69 (35.6\%) of respondents were classified as having good practices towards trachoma prevention and control with a mean score of $4.10 \pm 1.40$ SD (Table 4 ).

3.5. Factors Associated with Good Knowledge, Attitudes, and Practices towards Trachoma. Factors associated with good knowledge, attitudes, and practices towards trachoma
TABLE 1: Sociodemographic characteristics of study participants on knowledge, attitudes, and practices on trachoma in two rural districts of Tigray Region, Northern Ethiopia, 2017 ( $N=194)$.

\begin{tabular}{lcc}
\hline Variables & Numbers & Percent \\
\hline Age & & \\
$\quad$ 18-29 Years & 69 & 35.6 \\
30-49 Years & 87 & 44.8 \\
$\quad$ 250 Years & 38 & 19.6 \\
Marital status & & \\
$\quad$ Currently in union & 112 & 57.7 \\
$\quad$ Currently not in union** & 82 & 42.3 \\
Occupation & & \\
$\quad$ House wife & 180 & 92.8 \\
$\quad$ Others* & 14 & 7.2 \\
Educational status & & \\
$\quad$ Illiterate & 155 & 79.9 \\
$\quad$ Literate & 39 & 20.1 \\
Family size & & \\
$\quad \leq 5$ & 132 & 68 \\
$\quad>5$ & 62 & 32 \\
Monthly family income in US dollar & & 43.3 \\
$\quad<22$ USD & 84 & 44.8 \\
$\quad 22-44$ USD & 87 & 11.9 \\
$\quad>44$ USD & 23 &
\end{tabular}

* Others (government employed, merchant, and daily laborer). ${ }^{* *}$ Currently not in union (single, widowed, divorce, and separated).

prevention and control were observed. Having ever received health education about trachoma was significantly associated with good knowledge $(\mathrm{AOR}=4.10 ; 95 \% \mathrm{CI}: 1.91-8.79)$ and good attitudes (AOR $=2.10 ; 95 \% \mathrm{CI}: 1.02-4.25)$ (Tables 5 and 6). Moreover, good knowledge on trachoma was significantly associated with good practices towards trachoma prevention and control ( $\mathrm{AOR}=2.86$; 95\% CI: $1.46-5.62)$ after adjusting the other variables (Table 7 ).

\section{Discussion}

This study assessed knowledge, attitudes, and practices on trachoma in two districts of Tigray Region, Northern Ethiopia. Having ever received health education about trachoma was significantly associated with good knowledge and attitudes about trachoma prevention and control. Furthermore, good knowledge was significantly associated with good practices towards trachoma prevention and control.

In this study, $89.2 \%$ of respondents had ever heard about trachoma. This result was almost the same with the reports from Ethiopia (92.6\%) and Bangladesh (86\%), [17, 18]. However, it is higher than the report in Kenya (65.7\%) of participants had heard about trachoma [10]. The discrepancy could be due to the differences in social mobilization activities in different sites and studies methods.

The current study shows that $54.6 \%$ of respondents knew trachoma can be transmitted from person to person. The respondents mentioned that trachoma can be transmitted by contaminated fingers, flies, and contaminated towels. Similar studies from Kenya and Ethiopia reported that the most reported mode of trachoma transmission was contact with flies and dirty $[10,19]$. The current study results also indicate 
TABLE 2: Knowledge of study participants on trachoma in two rural districts of Tigray Region, Northern Ethiopia, 2017 ( $N=194)$.

\begin{tabular}{|c|c|c|}
\hline Variables & Numbers & Percent \\
\hline \multicolumn{3}{|l|}{ Heard about trachoma disease } \\
\hline Yes & 173 & 89.2 \\
\hline No & 21 & 10.8 \\
\hline \multicolumn{3}{|c|}{ Knew trachoma can be transmitted from person to person } \\
\hline Yes & 106 & 54.6 \\
\hline No & 88 & 45.4 \\
\hline \multicolumn{3}{|c|}{ Knew trachoma can be transmitted by contaminated fingers } \\
\hline Yes & 103 & 53.1 \\
\hline No & 91 & 46.9 \\
\hline \multicolumn{3}{|c|}{ Knew trachoma can be transmitted by flies } \\
\hline Yes & 69 & 35.6 \\
\hline No & 125 & 64.4 \\
\hline \multicolumn{3}{|c|}{ Knew trachoma can be transmitted by contaminated towels } \\
\hline Yes & 48 & 24.7 \\
\hline No & 146 & 75.3 \\
\hline \multicolumn{3}{|l|}{ Knew trachoma as preventable disease } \\
\hline Yes & 164 & 84.5 \\
\hline No & 35 & 18 \\
\hline \multicolumn{3}{|l|}{ Knew trachoma can lead to blindness } \\
\hline Yes & 161 & 83 \\
\hline No & 33 & 17 \\
\hline \multicolumn{3}{|c|}{ Knew trachoma can be prevented by washing hands with soap } \\
\hline Yes & 118 & 60.8 \\
\hline No & 76 & 39.2 \\
\hline \multicolumn{3}{|c|}{ Knew trachoma can be prevented by washing face } \\
\hline Yes & 131 & 67.5 \\
\hline No & 63 & 32.5 \\
\hline \multicolumn{3}{|c|}{ Knew trachoma can be prevented by using latrine } \\
\hline Yes & 37 & 19.1 \\
\hline No & 157 & 80.9 \\
\hline \multicolumn{3}{|c|}{$\begin{array}{l}\text { Knew trachoma can be prevented by improving environmental } \\
\text { sanitation }\end{array}$} \\
\hline Yes & 159 & 82 \\
\hline No & 30 & 15.5 \\
\hline \multicolumn{3}{|c|}{ Knew trachoma can be prevented by not using common towel } \\
\hline Yes & 48 & 24.7 \\
\hline No & 146 & 75.3 \\
\hline Mean + SD & $6.79 \pm 3.29$ & \\
\hline Median + IQR (interquartile range) & $7 \pm 5.25$ & \\
\hline \multicolumn{3}{|l|}{ Score of knowledge on trachoma } \\
\hline Good & 99 & 51 \\
\hline Poor & 95 & 49 \\
\hline
\end{tabular}

that the majority $(84.5 \%)$ of respondents knew trachoma as preventable disease. The participants responded that trachoma can be prevented by using latrine, improve environmental sanitation, not using common towel, and washing hand and face with soap. Having knowledge on washing face, not sharing towels, and environmental sanitation was important on prevention of trachoma infection [11].

Regarding attitudes and practices of respondents in the current study, the majority, $86.1 \%$, of respondents agreed that taking mass drug administration is important to prevent and control trachoma and $88.1 \%$ of the respondents took mass drug administration. This result is almost comparable with the other reports from Amhara and Tigray regions, and the coverage of mass drug administration ranged from
TABle 3: Attitudes of study participants on trachoma in two rural districts of Tigray Region, Northern Ethiopia, 2017 ( $N=194)$.

\begin{tabular}{lcc}
\hline Variables & Numbers & Percent \\
\hline Availability of adequate water is important for trachoma & \\
prevention and control & 185 & \\
Agree & 9 & 95.4 \\
Disagree & 134 & 4.6 \\
Personal hygiene is important for trachoma prevention & \\
Agree & 60 & 69.1 \\
Disagree & 171 & 30.9 \\
Latrine utilization is important for trachoma prevention & \\
Agree & 23 & 88.1 \\
Disagree & 167 & 11.9 \\
Taking mass drug administration is important to prevent and \\
control trachoma & 27 & 86.1 \\
Agree & $3.39 \pm 0.69$ & 13.9 \\
Disagree & $3 \pm 1$ & \\
Mean +SD & 96 & 49.5 \\
Median + IQR & 98 & 50.5 \\
Score of attitudes on trachoma prevention and control & \\
Good & & \\
Poor & &
\end{tabular}

TABle 4: Practice of community towards trachoma prevention and control in two rural districts of Tigray Region, Northern Ethiopia, $2017(N=194)$.

\begin{tabular}{lcc}
\hline Variables & Numbers & Percent \\
\hline Took mass drug administration for prevention of trachoma \\
Yes & 171 & 88.1 \\
No & 23 & 11.9 \\
Utilization of adequate water for bathing & \\
Yes & 150 & 77.3 \\
No & 44 & 22.7 \\
Having separated human and animal dwellings & \\
Yes & 149 & 76.8 \\
No & 45 & 23.2 \\
Latrine utilization & 72 & \\
Yes & 122 & 37.1 \\
No & 95 & 62.9 \\
Handwashing practice after using latrine & \\
Yes & 99 & 49 \\
No & 51 \\
Having proper solid waste disposal management & \\
Yes & 83 & 42.8 \\
No & 111 & 57.2 \\
Having proper liquid waste disposal management & \\
Yes & 15 & 7.7 \\
No & 179 & 92.3 \\
Having clean house compound & & \\
Yes & 59 & 30.4 \\
No & 135 & 69.6 \\
Mean +SD & $4.10 \pm 1.40$ & \\
Median + IQR & $4 \pm 2$ & \\
Score of practices on trachoma prevention and control & \\
Good & 69 & \\
Poor & 125 & \\
\hline
\end{tabular}

$76.8 \%$ to $93.3 \%$ [20-22]. On the other hand, $88.1 \%$ of respondents agreed that trachoma can be prevented by utilizing latrine. However, only $37.1 \%$ of households were utilizing a latrine. Our finding on latrine utilization was 
TABLE 5: Logistic regression analysis of selected variables with knowledge of study participants on trachoma in two rural districts of Tigray Region, Northern Ethiopia, 2017 ( $N=194)$.

\begin{tabular}{|c|c|c|c|c|}
\hline \multirow{2}{*}{ Variables } & \multicolumn{2}{|c|}{ Knowledge } & \multirow{2}{*}{ COR $(95 \% \mathrm{CI})$} & \multirow{2}{*}{ AOR $(95 \% \mathrm{CI})$} \\
\hline & Good (\%) & Poor $(\%)$ & & \\
\hline \multicolumn{5}{|l|}{ Marital status } \\
\hline Currently in union & $53(53.5)$ & $59(62.1)$ & 1 & 1 \\
\hline Currently not in union & $46(46.5)$ & $36(37.9)$ & $1.42(0.80-2.52)$ & $1.39(0.77-2.53)$ \\
\hline \multicolumn{5}{|c|}{ Availability of radio/television in the households } \\
\hline Yes & $19(19.2)$ & $12(12.6)$ & $1.64(0.75-3.60)$ & $1.35(0.60-3.03)$ \\
\hline No & $80(80.8)$ & $83(87.4)$ & 1 & 1 \\
\hline \multicolumn{5}{|c|}{ Ever received health education about trachoma } \\
\hline Yes & $88(88.9)$ & $62(65.3)$ & $4.26(2.00-9.07)^{* * *}$ & $4.10(1.91-8.79)^{* * *}$ \\
\hline No & $11(11.1)$ & $33(34.7)$ & 1 & 1 \\
\hline
\end{tabular}

${ }^{*}$ Statistically significant at $0.05<p<0.01 .{ }^{* *}$ Statistically significant at $0.01<p<0.001 .{ }^{* * *}$ Statistically significant at $p<0.001$.

TABLE 6: Logistic regression analysis of selected variables with attitude of study participants on trachoma in two rural districts of Tigray Region, Northern Ethiopia, $2017(N=194)$.

\begin{tabular}{|c|c|c|c|c|}
\hline \multirow{2}{*}{ Variables } & \multicolumn{2}{|c|}{ Attitude } & \multirow{2}{*}{ COR $(95 \% \mathrm{CI})$} & \multirow{2}{*}{ AOR $(95 \% \mathrm{CI})$} \\
\hline & Good (\%) & Poor (\%) & & \\
\hline \multicolumn{5}{|l|}{ Age } \\
\hline $18-29$ years & $30(31.2)$ & $39(39.8)$ & 1 & 1 \\
\hline $30-49$ years & $49(51)$ & $38(38.8)$ & $1.68(0.89-3.17)$ & $1.46(0.75-2.84)$ \\
\hline$\geq 50$ years & $17(17.3)$ & $21(21.4)$ & $1.10(0.47-2.34)$ & $1.20(0.53-2.72)$ \\
\hline \multicolumn{5}{|l|}{ Family size } \\
\hline$\leq 5$ & $59(61.5)$ & $73(74.5)$ & 1 & 1 \\
\hline$>5$ & $37(38.5)$ & $25(25.5)$ & $1.83(0.99-3.38)$ & $1.52(0.78-2.93)$ \\
\hline \multicolumn{5}{|c|}{ Ever received health education about trachoma } \\
\hline Yes & $81(84.4)$ & $69(70.4)$ & $2.27(1.13-4.58)^{*}$ & $2.10(1.02-4.25)^{*}$ \\
\hline No & $15(15.6)$ & $29(29.6)$ & 1 & 1 \\
\hline
\end{tabular}

${ }^{*}$ Statistically significant at $0.05<p<0.01 .{ }^{* *}$ Statistically significant at $0.01<p<0.001 .{ }^{* * *}$ Statistically significant at $p<0.001$.

lower than from previous studies in Ethiopia $[9,17]$. This difference could be due to differences in the study areas because our study was not included urban communities. Latrine utilization has been considered an important intervention for trachoma prevention and control [23]. It is important to reduce burden of flies and improve environmental sanitation through proper disposal of human excreta [24].

In the current study, health education about trachoma was significantly associated with knowledge on trachoma. Previous interventional studies from Ethiopia and Egypt reported that mothers' knowledge was significantly improved after the implementation of the program based health education $[16,25]$. Providing health education about trachoma can increase knowledge on trachoma dramatically [26]. With adequate and appropriate health education, communities may increase knowledge on the cause of trachoma, how it is transmitted, and how the disease is prevented [2].

Health education on trachoma was also a predictor of attitudes on trachoma prevention and control. The odds of good attitudes on trachoma prevention and control among participants who had ever received health education were 2.1 times higher than participants who had not ever received health education about trachoma. Health education can help reducing misconceptions and changing cultural perceptions of communities on trachoma prevention and control $[11,27]$. Effective health education is the key to building the favorable attitudes on trachoma prevention and control among communities to achieve the goal of eliminating trachoma as a public health problem [28, 29]. In Ethiopia, health extension workers and WDG leaders are giving health education to communities on personal hygiene, environmental sanitation, and mass drug administration for prevention and control of trachoma [22]. This could be the possible explanation that if mothers have received health education on trachoma, they may feel responsibility and accountability to prevent their children and families from trachoma infection. This could be resulted to have good attitudes on prevention and control of trachoma.

The current study revealed that mothers who have good knowledge on trachoma was significantly associated with good practices on trachoma prevention and control. This is supported by a study from Egypt, reported that knowledge was significantly associated with trachoma prevention and control practices [25]. Another study from Ethiopia also revealed that good knowledge was a predictor of good practices towards childhood blindness prevention [19]. Increasing knowledge of trachoma transmission and prevention (e.g., F\&E-related preventive behaviors) is important to improve practices of trachoma prevention and control [30]. This might be due to the reason that if someone 
TABLE 7: Logistic regression analysis of selected variables with practice of communities on trachoma in two rural districts of Tigray Region, Northern Ethiopia, $2017(N=194)$.

\begin{tabular}{|c|c|c|c|c|}
\hline \multirow{2}{*}{ Variables } & \multicolumn{2}{|c|}{ Practice } & \multirow{2}{*}{ COR $(95 \% \mathrm{CI})$} & \multirow{2}{*}{ AOR (95\% CI) } \\
\hline & Good (\%) & Poor $(\%)$ & & \\
\hline \multicolumn{5}{|l|}{ Marital status } \\
\hline Currently in union & $33(47.8)$ & $79(63.2)$ & 1 & 1 \\
\hline Currently not in union & $36(52.2)$ & $46(36.8)$ & $1.87(1.03-3.40)^{*}$ & $1.71(0.88-3.23)$ \\
\hline \multicolumn{5}{|l|}{ Educational status } \\
\hline Illiterate & $52(75.4)$ & $103(82.4)$ & 1 & 1 \\
\hline Literate & $17(24.6)$ & $22(17.6)$ & $1.53(0.75-3.13)$ & $1.01(0.43-2.40)$ \\
\hline \multicolumn{5}{|l|}{ Occupation } \\
\hline House wife & $61(88.4)$ & $119(95.2)$ & 1 & 1 \\
\hline Others & $8(11.6)$ & $6(4.8)$ & $2.60(0.86-7.83)$ & $2.10(0.57-7.64)$ \\
\hline \multicolumn{5}{|c|}{ Availability of radio/television in the household } \\
\hline Yes & $18(26.1)$ & $13(10.4)$ & $3.04(1.39-6.68)^{* *}$ & $2.20(0.93-5.20)$ \\
\hline No & $51(73.9)$ & $112(89.6)$ & 1 & 1 \\
\hline \multicolumn{5}{|c|}{ Time taken to fetch water from its source } \\
\hline$\leq 30$ & $57(82.6)$ & $94(75.2)$ & $1.57(0.75-3.29)$ & $0.97(0.40-2.40)$ \\
\hline$>30$ minutes & $12(17.4)$ & $31(24.8)$ & 1 & 1 \\
\hline \multicolumn{5}{|c|}{ Access to sustainable sufficient water supply } \\
\hline Yes & $63(91.3)$ & $104(83.2)$ & $2.12(0.81-5.54)$ & $2.02(0.66-6.18)$ \\
\hline No & $6(8.7)$ & $21(16.8)$ & 1 & 1 \\
\hline \multicolumn{5}{|c|}{ Ever received health education about trachoma } \\
\hline Yes & $60(87)$ & & $2.59(1.16-5.78)^{*}$ & $1.55(0.63-3.83)$ \\
\hline No & $9(13)$ & & 1 & 1 \\
\hline \multicolumn{5}{|c|}{ Total score of knowledge on trachoma } \\
\hline Good & $22(31.9)$ & $73(58.4)$ & $3.0(1.62-5.57)^{* *}$ & $2.86(1.46-5.62)^{* *}$ \\
\hline Poor & $47(68.1)$ & $52(41.6)$ & 1 & 1 \\
\hline \multicolumn{5}{|c|}{ Total score of attitudes on trachoma } \\
\hline Good & $30(43.5)$ & $68(54.4)$ & $1.55(0.86-2.80)$ & $1.51(0.79-2.87)$ \\
\hline Poor & $39(56.5)$ & $57(45.6)$ & 1 & 1 \\
\hline
\end{tabular}

${ }^{*}$ Statistically significant at $0.05<p<0.01 .{ }^{* *}$ Statistically significant at $0.01<p<0.001 .{ }^{* * *}$ Statistically significant at $p<0.001$.

knew benefit of personal hygiene and environmental sanitation, she/he could practice to prevent and control trachoma [1].

4.1. Limitation of the Study. The sample size of this study was relatively small and did not consider the design effect to account for the multilevel nature of the sampling design. This may have affected both the precision of the prevalence estimates as well as the statistical inferences of the modeling. Responses on knowledge-, attitude-, and practice-related questions might be influenced by social desirability biases. Correct responses for those are socially acceptable opinions and practices, and incorrect responses for those are socially undesirable opinions and practices. There might be missed some factors that could influence knowledge, attitudes, and practices that were not considered in the model.

\section{Conclusion and Recommendation}

Our study implies that areas with high burden of trachoma need to improve communities' knowledge, attitudes, and practices on trachoma prevention and control in order to eliminate trachoma as a public health problem. Therefore, health education focused on the SAFE strategy should be provided at community level, during mass drug administration, and at health facilities while people are waiting to receive treatment to increase good knowledge and changing cultural perceptions that contribute to behavioral changing and increase good practices towards trachoma prevention and control among communities.

\section{Abbreviations}

AOR: Adjusted odds ratio

CI: Confidence interval

COR: Crude odds ratio

SD: $\quad$ Standard deviation

WDG: Women developmental group.

\section{Data Availability}

The datasets used and/or analyzed during the current study are available from the corresponding author on reasonable request.

\section{Conflicts of Interest}

All authors declare that they have no conflicts of interest.

\section{Authors' Contributions}

HG, NM, TT, KF, TG, AB, FG, and KD contributed to the conception, development of data collection tools, design, data collection, analysis, and interpretation of the data. HG 
did the major writing work of the manuscript and revised the draft and final manuscript. NM, TT, KF, TG, AB, FG, and KD revised the draft of the manuscript. All authors read and approved the final manuscript.

\section{Acknowledgments}

The authors gratefully acknowledge Light for the World for financial support. The authors acknowledge the Tigray Regional Health Bureau and Raya-Alamata and Endamekoni district health offices for providing technical and logistic supports. The authors also thank the supervisors, data collectors, and study participants for giving them paramount information.

\section{References}

[1] P. Emerson, L. Frost, D. Mabey, and R. Bailey, "Implementing the safe strategy for trachoma control: a toolbox of interventions for promoting facial cleanliness and environmental improvement," vol. 44, 2006, https://www.cartercenter.org/ documents/2302.

[2] The Carter Center, Women and Trachoma: Achieving Gender Equity in the Implementation of SAFE, The Carter Center, Atlanta, GA, USA, 2009.

[3] N. J. Allen, In Practice Eliminating Blindness from Trachoma Infection Lessons Learned from the Conrad N, Hilton Foundation, Westlake Village, CA, USA, 2012.

[4] WHO and Weekly Epidemiological Record, "WHO alliance for the global elimination of trachoma by 2020," Progress Report on Elimination of Trachoma, no. 29, pp. 317-328, 2019.

[5] WHO, Eliminating trachoma: WHO Announces Sustained Progress with Hundreds of Millions of People No Longer at Risk of Infection, News release, Maputo, Geneva, 2019, https:// www.who.int/news-room/detail/27-06-2019-eliminatingtrachoma-who-announces-sustained-progress-withhundreds-of-millions-of-people-no-longer-at-risk-ofinfection.

[6] F. M. Altherr, A. W. Nute, M. Zerihun et al., "Associations between water, sanitation and hygiene (wash) and trachoma clustering at aggregate spatial scales, amhara, Ethiopia," Parasites and Vectors, vol. 12, p. 540, 2019.

[7] M. E. Stocks, S. Ogden, D. Haddad, D. G. Addiss, C. McGuire, and M. C. Freeman, "Effect of water, sanitation, and hygiene on the prevention of trachoma: a systematic review and metaanalysis," PLoS Med, vol. 11, no. 2, 2014.

[8] B. Tadesse, A. Worku, A. Kumie, and S. A. Yimer, "The burden of and risk factors for active trachoma in the North and South Wollo Zones of Amhara Region, Ethiopia: a crosssectional study," Infectious Diseases of Poverty, vol. 6, p. 143, 2017.

[9] K. Ketema, M. Tiruneh, D. Woldeyohannes, and D. Muluye, "Active trachoma and associated risk factors among children in baso liben district of east gojjam, Ethiopia," BMC Public Health, vol. 12, p. 1105, 2012.

[10] P. N. Munguti, L. Ng'ang'a, and J. Muttunga, "Knowledge, practices and perception on trachoma and its influence on health seeking behaviour of the pastoralist patients in kajiado central division, Kenya," East and Central Africa Medical Journal, vol. 2, pp. 89-96, 2015.

[11] D. W. Njomo, J. Karimurio, G. O. Odhiambo et al., "Knowledge, practices and perceptions of trachoma and its control among communities of Narok County, Kenya,"
Tropical Diseases, Travel Medicine and Vaccines, vol. 2, p. 13, 2016.

[12] I. Golovaty, L. Jones, B. Gelaye et al., "Access to water source, latrine facilities and other risk factors of active trachoma in Ankober, Ethiopia," PLoS One, vol. 4, no. 8, 2009.

[13] C. Mpyet, M. Goyol, and C. Ogoshi, "Personal and environmental risk factors for active trachoma in children in Yobe state, north-eastern Nigeria," Tropical Medicine \& International Health, vol. 15, no. 2, pp. 168-172, 2010.

[14] T. Ayalew, "Prevalence of trachoma and associated factors of children aged 1-9 Years in community led total sanitation and hygiene triggered village and none triggered in girar jarso woreda, north shoa, oromia, Ethiopia," IJIR, vol. 2, no. 9, pp. 1444-1455, 2016.

[15] S. T. Sherief, C. Macleod, G. Gigar et al., "The prevalence of trachoma in Tigray region, northern Ethiopia: results of 11 population-based prevalence surveys completed as part of the global trachoma mapping project," Ophthalmic Epidemiology, vol. 23, no. sup1, pp. 94-99, 2016.

[16] P. Cumberland, T. Edwards, G. Hailu et al., "The impact of community level treatment and preventative interventions on trachoma prevalence in rural Ethiopia," International Journal of Epidemiology, vol. 37, no. 3, pp. 549-558, 2008.

[17] K. Mengistu, M. Shegaze, K. Woldemichael, H. Gesesew, and Y. Markos, "Prevalence and factors associated with trachoma among children aged 1-9 years in zala district, gamo gofa zone, southern Ethiopia," Clinical Ophthalmology, vol. 10, pp. 1663-1670, 2016.

[18] F. M. A. Islam, R. Chakrabarti, S. Z. Islam, R. P. Finger, and C. Critchley, "Factors associated with awareness, attitudes and practices regarding common eye diseases in the general population in a rural district in Bangladesh: the Bangladesh population-based diabetes and eye study (BPDES)," PLoS One, vol. 10, no. 7, 2015.

[19] W. T. Belaynew, M. Z. Berihun, A. A. Tadesse, and A. W. Yared, "Knowledge and practice on childhood blindness among communities in Northwest Ethiopia: implications to blindness prevention programs," Journal of Ophthalmology, 2013.

[20] T. Astale, E. Sata, M. Zerihun et al., "Population-based coverage survey results following the mass drug administration of azithromycin for the treatment of trachoma in Amhara, Ethiopia," PLoS Neglected Tropical Diseases, vol. 12, no. 2, 2018.

[21] C. D. Ebert, T. Astale, E. Sata et al., "Population coverage and factors associated with participation following a mass drug administration of azithromycin for trachoma elimination in Amhara, Ethiopia," Tropical Medicine \& International Health, vol. 24, no. 4, pp. 493-501, 2019.

[22] A. Mulugeta, G. B. Gebregergs, S. Asfaw et al., "Coverage, social mobilization and challenges of mass zithromax administration campaign in south and south east zones of Tigray, Northern Ethiopia: a cross sectional study," PLoS Neglected Tropical Diseases, vol. 12, no. 2, 2018.

[23] M. Haile, B. D. Gaynor, Z. Tadesse et al., "The association between latrine use and trachoma: a secondary cohort analysis from a randomized clinical trial," The American Journal of Tropical Medicine and Hygiene, vol. 89, no. 4, pp. 717-720, 2013.

[24] J. O. Okullo, W. N. Moturi, and G. M. Ogendi, "Open defaecation and its effects on the bacteriological quality of drinking water sources in isiolo county, Kenya," Environ Health Insights, vol. 11, pp. 1-8, 2017. 
[25] E. A. Awad, B. zatgowayed, L. M. Fouda, H. A. Ebrahim, and S. Elsayed, "Effectiveness of health education program on rural mothers' knowledge and practice regarding blinding trachoma among their children," IOSR Journal of Nursing and Health Science (IOSR-JNHS), vol. 7, pp. 88-99, 2018.

[26] R. A. Rubinstein and S. D. Lane, "Articles from intervention to Outcome: the relationship between knowledge and behavior in a trachoma control project," Journal of Healthcare Science and The Humanities, vol. 2, no. 2, 2012.

[27] D. Ramai, R. Elliott, S. Goldin, and T. Pulisetty, "A crosssectional study of pediatric eye care perceptions in Ghana, Honduras, and India," Journal of Epidemiology and Global Health, vol. 5, no. 2, pp. 133-142, 2015.

[28] M. Zondervan, H. Kuper, A. Solomon, and J. Buchan, "Health promotion for trachoma control," Community Eye Heal, vol. 17, no. 52, pp. 57-58, 2004.

[29] K. Thompson, H. Hutchins, A. Baio et al., "Health beliefs and perceptions of trachoma in communities on the bijagos archipelago of Guinea bissau," Ophthalmic Epidemiology, vol. 22, no. 3, pp. 190-199, 2015.

[30] M. G. Delea, H. Solomon, A. W. Solomon, and M. C. Freeman, "Interventions to maximize facial cleanliness and achieve environmental improvement for trachoma elimination: a review of the grey literature," PLoS Neglected Tropical Diseases, vol. 12, no. 1, pp. 1-26, 2018. 